\title{
Electron and hole spectra in the superlattice of cylindrical quantum wires
}

\author{
M.V.Tkach ${ }^{1}$, I.V.Pronyshyn ${ }^{1}$, A.M.Makhanets ${ }^{1}$, \\ G.G.Zegrya ${ }^{2}$, V.N.Golovach ${ }^{2}$ \\ 1 Chernivtsi State University, \\ 2 Kotsiubinsky Str., 274012 Chernivtsi, Ukraine \\ 2 A.F.loffe Physical-Technical Institute, \\ 194021 St.Petersburg, Russia
}

Received June 26, 1998

\begin{abstract}
Electron and hole spectra in $\beta$-HgS cylindrical quantum wire superlattice in $\beta$-CdS matrix are calculated within the method of augmented plane waves. The energy term determined by the movement of quasiparticles in direction perpendicular to the axial axis of the wire is presented by the alternating zones with a positive and a negative effective mass. The degeneration on the magnetic quantum number outside $\Gamma$ point of Brillouin zone is taken off when the potential of the superlattice quantum wires is taken into account. The ground zones energy dependence of plane quasiparticle movement on the radii of the quantum wires as well as the distance between the wires are investigated.
\end{abstract}

Key words: semiconductor, electron, hole, superlattice, quantum wire

PACS: $79.60 . j \mathrm{v}$

\section{Introduction}

The new unusual phenomena of fundamental character and exclusive possibilities of nanoheterosystem practical utilization are of great interest to the researchers. In [1], the prospects for a further development of this branch were recently analyzed. Among the others, the technologically produced superlattice quantum wells (QW) were pointed out. Changing their spatial characteristics one can control the fundamental properties of these systems (location of energy zones, quasiparticle effective masses, etc.).

One of the objects of the present research is to provide a heterosystem consisting of quantum wires (QW) of one material embedded into the other material and forming the superlattice in the direction perpendicular to the axial axis of QW. 
When the QW length is much bigger than the length of quasiparticle free movement then the QW can be assumed to be infinitely long. The QW radius and the distance between the nearest neighbours are of nanosize. It is clear that the spatial changing of QW sizes and locations should bring about the change of electron, hole and exciton zones in such a superlattice.

The purpose of this work is to perform theoretical calculations of electron and hole spectra and to define the wave functions of these quasiparticles in the cylindrical superlattice of quantum wires (CSQW).

\section{The theory of electron (hole) spectra in CSQW}

The system consisting of cylindrical QW (semiconductor material 1) periodically located in the medium (semiconductor material 2) is under study. For the sake of simplicity we observe an electron assuming that in a cylindrical coordinate system with $\mathrm{OZ}$ axis along the axial axis of one QW, the potential energy and the effective mass are different in different media

$$
\begin{aligned}
U(\vec{r}) & = \begin{cases}-U_{0} ; & \text { in QW } \\
0 ; & \text { outside of QW, }\end{cases} \\
\mu(\vec{r}) & = \begin{cases}\mu_{1} ; & \text { in QW } \\
\mu_{2} ; & \text { outside of QW. }\end{cases}
\end{aligned}
$$

In order to obtain spectrum and electron wave functions one has to solve Schrödinger equation with Hamiltonian which in a cylindrical coordinate system has the following form

$$
\hat{H}=-\frac{\hbar^{2}}{2}\left(\vec{\nabla}_{\rho \phi} \frac{1}{\mu(\rho, \phi)} \vec{\nabla}_{\rho \phi}+\frac{1}{\mu(\rho, \phi)} \frac{\partial^{2}}{\partial z^{2}}\right)+U(\rho) .
$$

It is impossible to part $\mathrm{z}$-th variable exactly in a Schrödinger equation due to the dependence of $\mu$ on $\rho$ in (2). Since it is suitable to introduce the "average" (constant) electron effective mass (e.q. $\mu_{\|}^{0}=\frac{\mu_{1}+\mu_{2}}{2}$ ) and represent Hamiltonian (3) as

$$
\hat{H}=\hat{H}_{0}+\Delta \hat{H}
$$

where

$$
\hat{H}_{0}=-\frac{\hbar^{2}}{2}\left(\vec{\nabla}_{\rho \phi} \frac{1}{\mu(\rho, \phi)} \vec{\nabla}_{\rho \phi}+\frac{1}{\mu(\rho, \phi)} \frac{\partial^{2}}{\partial z^{2}}\right)+U(\rho)
$$

is a basic part and

$$
\Delta \hat{H}=\frac{\hbar^{2}}{2}\left(\frac{1}{\mu_{\|}^{0}}-\frac{1}{\mu(\rho)}\right) \frac{\partial^{2}}{\partial z^{2}}
$$

is the perturbation.

Now in the equation

$$
\hat{H}_{0} \psi^{0}(\rho, \phi, z)=E^{0} \psi^{0}(\rho, \phi, z)
$$



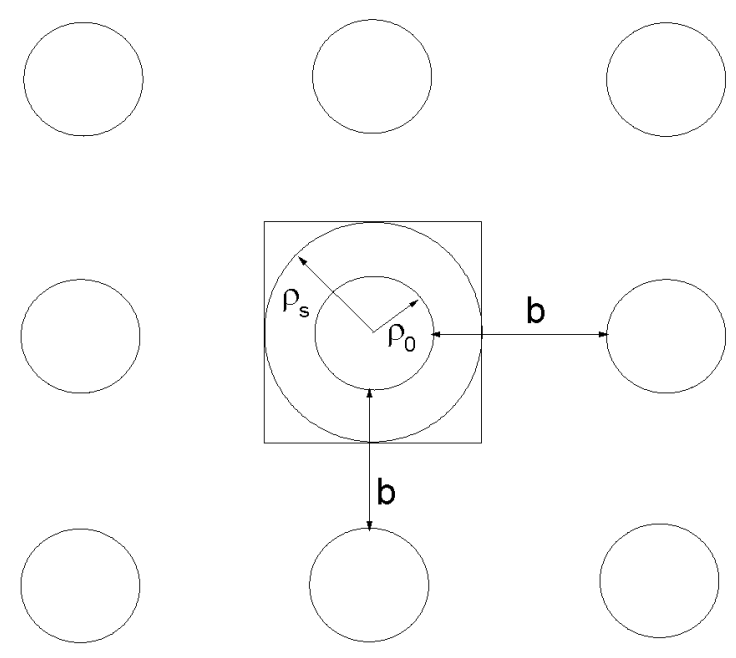

Figure 1. CSQW geometry

z-th component can be parted. Really, representing $\psi^{0}(\rho, \phi, z)$ in the form

$$
\psi^{0}(\rho, \phi, z)=\frac{1}{\sqrt{L}} \psi^{0}(\vec{\rho}) \mathrm{e}^{\mathrm{i} k_{\|} z}
$$

where $L$ is the length of the basic region along the QW axis, one can get

$$
E^{0}=E_{\perp}^{0}+E^{0}\left(k_{\|}\right)=E_{\perp}^{0}+\frac{\hbar^{2} k_{\|}^{2}}{2 \mu_{\|}^{0}}
$$

where $E_{\perp}^{0}$ value and $\psi^{0}(\vec{\rho})$ wave function are defined by the Schrödinger equation

$$
\left[-\frac{\hbar^{2}}{2}\left(\frac{1}{\rho} \frac{\partial}{\partial \rho} \rho \frac{1}{\mu(\rho, \phi)} \frac{\partial}{\partial \rho}+\frac{1}{\rho^{2}} \frac{\partial^{2}}{\partial \phi^{2}}\right)+U(\rho)\right] \psi^{0}(\vec{\rho})=E_{\perp}^{0} \psi^{0}(\vec{\rho}) .
$$

The latter can be solved using the augmented plane waves (APW) method which is well known in the three dimensional systems [2]. Modification of APW method for the case of the system under research is performed in the following way. Let us set the beginning of the plane system coordinate into the center of the circle with $\rho_{0}$ radius (figure 1 ), so that it coincides with the node of the direct lattice. Within one Vigner-Zeit cell the so called "m-t potential" has the simple form

$$
U(\vec{\rho})= \begin{cases}-U_{0} ; & \rho \leqslant \rho_{0} \\ 0 ; & \rho>\rho_{0}\end{cases}
$$

According to the APW method, the Schrödinger equation for the region of the plane space inside the quantum wells where the wave function is presented as a superposition of cylindrical harmonics is solved exactly.

As the potential is equal to zero in the space outside the wells, the wave function should have the form of the plane wave which can be expanded into a series by the 
cylindrical harmonics. The expanding coefficients can be obtained from the condition of function continuity on the border of the circle of $\rho_{0}$ radius. In such a way the trial wave function is found in the form of augmented plane wave (APW).

$$
\Phi_{\vec{k}_{\perp}-\vec{g}}= \begin{cases}\frac{1}{\sqrt{\Omega_{0}}} \sum_{-\infty}^{\infty} \mathrm{i}^{m} \frac{J_{m}\left(\left|\vec{k}_{\perp}-\vec{g}\right| \rho_{0}\right)}{J_{m}\left(\alpha \rho_{0}\right)} J_{m}(\alpha \rho) \mathrm{e}^{\mathrm{i} m\left(\phi-\phi_{\vec{k}_{\perp}-\vec{g}}\right)} ; & \rho \leqslant \rho_{0}, \\ \frac{1}{\sqrt{\Omega_{0}}} \mathrm{e}^{\mathrm{i}\left(\vec{k}_{\perp}-\vec{g}\right) \vec{\rho}}=\frac{1}{\sqrt{\Omega_{0}}} \sum_{-\infty}^{\infty} \mathrm{i}^{m} J_{m}\left(\left|\vec{k}_{\perp}-\vec{g}\right| \rho\right) \mathrm{e}^{\mathrm{i} m\left(\phi-\phi_{\vec{k}_{\perp}-\vec{g}}\right)} ; & \rho>\rho_{0},\end{cases}
$$

where $\Omega_{0}$ - volume of unit cell, $J_{m}$ - Bessel cylindrical function, $m$ - magnetic quantum number, $\vec{k}_{\perp}$ - quasiparticle wave vector, $\vec{g}$ - vector of the reverse lattice, $\left|\vec{k}_{\perp}-\vec{g}\right|$ and $\phi_{\vec{k} i_{\perp}-\vec{g}}-$ polar coordinates of $\vec{k}_{\perp}-\vec{g}$ vector, $\alpha=\hbar^{-1} \sqrt{2 \mu_{1}\left(U_{0}-E_{\perp}\right)}$.

APW (12) satisfies the Bloch periodical condition but does not satisfy the Schrödinger equation with the potential of all the superlattice because the relationship between the energy and wave vector has not been accounted for by now. In order to find this relationship according to the Bloch theorem one can find the quasiparticle wave function as a linear combination of the augmented plane waves

$$
\psi_{\vec{k}_{\perp}}^{0}(\vec{\rho})=\sum_{\vec{g}} c_{\vec{k}_{\perp-\vec{g}}} \Phi_{\vec{k}_{\perp}-\vec{g}}(\vec{\rho}),
$$

where the summing is performed over the vectors of the reverse lattice and $c_{\vec{k}-\vec{g}}$ coefficients are to be defined.

It is better to use the variational method instead of the Schrödinger equation because every APW has the rupture of the derivative on the border between the quantum wells and interwell regions. Let us define the energy functionals on the wave functions (13)

$$
\Lambda=\int_{\Omega_{0}}\left\{\frac{\hbar^{2}}{2} \vec{\nabla}_{\rho, \phi} \psi_{\vec{k}_{\perp}}^{0 *} \frac{1}{\mu(\rho, \phi)} \vec{\nabla}_{\rho, \phi} \psi_{\vec{k}_{\perp}}^{0}+\left(U(\rho)-E_{\perp}^{0}\right) \psi_{\vec{k}_{\perp}}^{0 *} \psi_{\vec{k}_{\perp}}^{0}\right\} \mathrm{d} \vec{\rho} .
$$

Minimization of $\Lambda(\delta \Lambda=0)$ functional over $c_{\vec{k}-\vec{g}}$ leads to the system of equations

$$
\left\{\frac{\hbar^{2}}{2 \mu_{2}}\left(\vec{k}_{\perp}-\vec{g}\right)^{2}-E_{\perp}^{0}\right\} c_{\vec{k}_{\perp}-\vec{g}}+\sum_{\vec{g}^{\prime}} \Gamma_{\vec{g} \vec{g}^{\prime}} c_{\vec{k}-\vec{g}^{\prime}}=0,
$$

where $\Gamma_{\vec{g} \vec{g}^{\prime}}$ is the integral containing $\Phi_{\vec{k}_{\perp}-\vec{g}} \Phi_{\vec{k}_{\perp}-\vec{g}^{\prime}}$ functions and Hamiltonian with periodical potential of the system. Integrating in $\Gamma_{\vec{g} \vec{g}^{\prime}}$ over the unit cell volume $\Omega_{0}$ we obtain

$$
\begin{gathered}
\Gamma_{\vec{g} \vec{g}^{\prime}}=\frac{2 \pi \rho_{0}}{\Omega_{0}}\left\{-\left[\frac{\hbar^{2}}{2 \mu 2}\left(\vec{k}_{\perp}-\vec{g}\right)\left(\vec{k}_{\perp}-\vec{g}^{\prime}\right)-E_{\perp}^{0}\right] \frac{J_{1}\left(\left|\vec{g}-\vec{g}^{\prime}\right| \rho_{0}\right)}{\left|\vec{g}-\vec{g}^{\prime}\right|}\right. \\
\left.+\frac{\hbar^{2}}{2 \mu_{1}} \sum_{m=-\infty}^{\infty} \mathrm{e}^{\mathrm{i} m \phi_{\vec{g} \vec{g}^{\prime}}} J_{m}\left(\left|\vec{k}_{\perp}-\vec{g}\right| \rho_{0}\right) J_{m}\left(\left|\vec{k}_{\perp}-\vec{g}^{\prime}\right| \rho_{0}\right)\left[\frac{\mathrm{d}}{\mathrm{d} \rho} \ln J_{m}(\alpha \rho)\right]_{\rho=\rho_{0}}\right\},
\end{gathered}
$$

where $\phi_{\vec{g} \vec{g}^{\prime}}$ is the angle between $\left(\vec{k}_{\perp}-\vec{g}\right)$ and $\left(\vec{k}_{\perp}-\vec{g}^{\prime}\right)$ vectors. The first term in the right side of (16) arises due to the regions outside the quantum wells and the rest 
of the terms arise due to the impact of the gradient operator into the augmented plane waves. $\Gamma_{\vec{g} \vec{g}^{\prime}}$ values are the Fourier-components of the effective potential of the superlattice quantum wells.

The condition of the non-trivial solution of equation (15) leads to the secular equation

$$
\operatorname{det}\left|\left[\frac{\hbar^{2}}{2 \mu_{2}}\left(\vec{k}_{\perp}-\vec{g}\right)^{2}-E_{\perp}^{0}\right] \delta_{\vec{g} \vec{g}^{\prime}}+\Gamma_{\vec{g} \vec{g}^{\prime}}\right|=0,
$$

which determines the quasiparticle energy spectrum $E^{0}\left(\vec{k}_{\perp}\right)$ due to its movement in the plane $\perp$ to the axial axis of QW. Generally, the equation (15) hasn't got just one but several $(\tau)$ solutions when $\vec{k}_{\perp}$ is fixed. Then, the corresponding energies are further denoted as $E_{n=1,2, \ldots, \tau}^{0}\left(\vec{k}_{\perp}\right)$.

Now according to (9) the electron energy spectrum in the superlattice QW in zeroth approximation $\left(\right.$ on $\left.\mu_{\| \mid}^{0}\right)$ is

$$
E_{n}^{0}\left(\vec{k}_{\perp}, k_{\|}\right)=E_{n}^{0}\left(\vec{k}_{\perp}\right)+\frac{\hbar^{2} k_{\|}^{2}}{2 \mu_{\|}^{0}} .
$$

From the conditions of normalization

$$
\sum_{\vec{g} \vec{g}^{\prime}} c_{\vec{k}_{\perp}-\vec{g}}^{*} c_{\vec{k}_{\perp}-\vec{g}^{\prime}}\left(P_{\vec{g} \vec{g}^{\prime}}+P_{\vec{g} \vec{g}^{\prime}}\right)=1
$$

where the values

$$
\begin{gathered}
P_{\vec{g} \vec{g}^{\prime}}=\delta_{\vec{g} \vec{g}^{\prime}}-\frac{2 \pi \rho_{0}}{\Omega_{0}} \frac{J_{1}\left(\left|\vec{g}-\vec{g}^{\prime}\right| \rho_{0}\right)}{\left|\vec{g}-\vec{g}^{\prime}\right|} \\
S_{\vec{g} \vec{g}^{\prime}}=\frac{\pi \rho_{0}^{2}}{\Omega_{0}} \sum_{m=-\infty}^{\infty} J_{m}\left(\left|\vec{k}_{\perp}-\vec{g}\right| \rho_{0}\right) J_{m}\left(\left|\vec{k}_{\perp}-\vec{g}^{\prime}\right| \rho_{0}\right) \frac{J_{m}^{2}\left(\alpha \rho_{0}\right)-J_{m-1}\left(\alpha \rho_{0}\right) J_{m+1}\left(\alpha \rho_{0}\right)}{J_{m}^{2}\left(\alpha \rho_{0}\right)}
\end{gathered}
$$

and the system of equations (15) $c_{n \vec{k}_{\perp}-\vec{g}}^{0}$ coefficients can be found and since the electron wave functions are defined as

$$
\psi_{n}^{0}(\vec{\rho}, z)=\frac{1}{\sqrt{L}} \mathrm{e}^{\mathrm{i} k_{||} z} \sum_{\vec{g}} c_{n, \vec{k}_{\perp-\vec{g}}} \Phi_{n, \vec{k}_{\perp-\vec{g}}}(\vec{\rho})
$$

in the superlattice QW in zeroth approximation (on effective mass).

Taking into account perturbation (6) and wave functions (13), we can obtain the longitudinal effective mass in the first approximation

$$
\mu_{\| n}^{(1)}=\frac{\mu_{\| \mid}^{0}}{1+I_{n} \mu_{\|}^{0}}
$$

where

$$
\begin{aligned}
I_{n}= & \sum_{\vec{g} \vec{g}^{\prime}} c_{n, \vec{k}_{\perp}-\vec{g}}^{*} c_{n, \vec{k}_{\perp}-\vec{g}^{\prime}} \int_{\Omega_{0}}\left(\frac{1}{\mu(\rho)}-\frac{1}{\mu_{\|}^{0}}\right) \Phi_{n, \vec{k}_{\perp}-\vec{g}}^{*}(\vec{\rho}) \Phi_{n, \vec{k}_{\perp}-\vec{g}^{\prime}}(\vec{\rho}) \mathrm{d} \vec{\rho} \\
& =\sum_{\vec{g} \vec{g}^{\prime}} c_{n, \vec{k}_{\perp}-\vec{g}}^{*} c_{n, \vec{k}_{\perp}-\vec{g}^{\prime}}\left\{\left(\frac{1}{\mu_{2}}-\frac{1}{\mu_{\|}^{0}}\right) P_{\vec{g} \vec{g}^{\prime}}+\left(\frac{1}{\mu_{1}}-\frac{1}{\mu_{\|}^{0}}\right) S_{\vec{g} \vec{g}^{\prime}} .\right.
\end{aligned}
$$




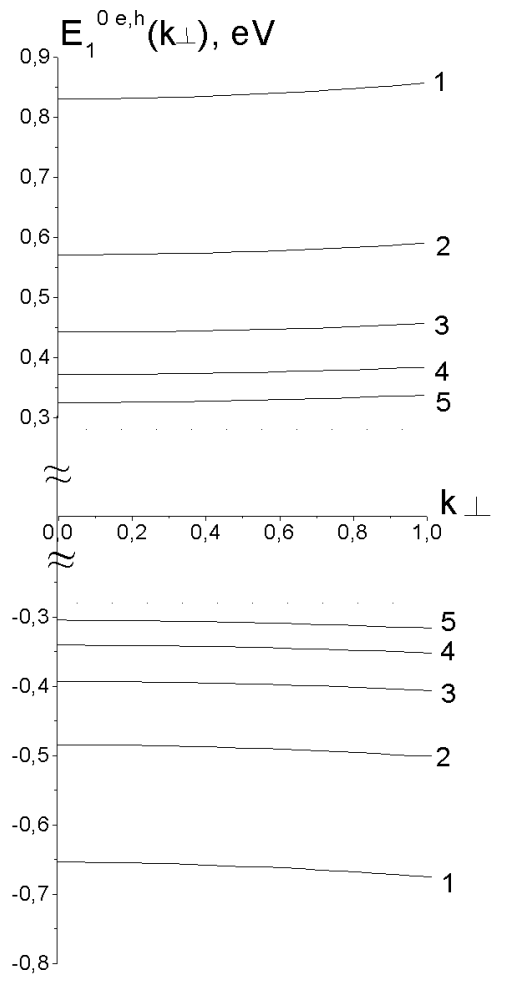

(a)

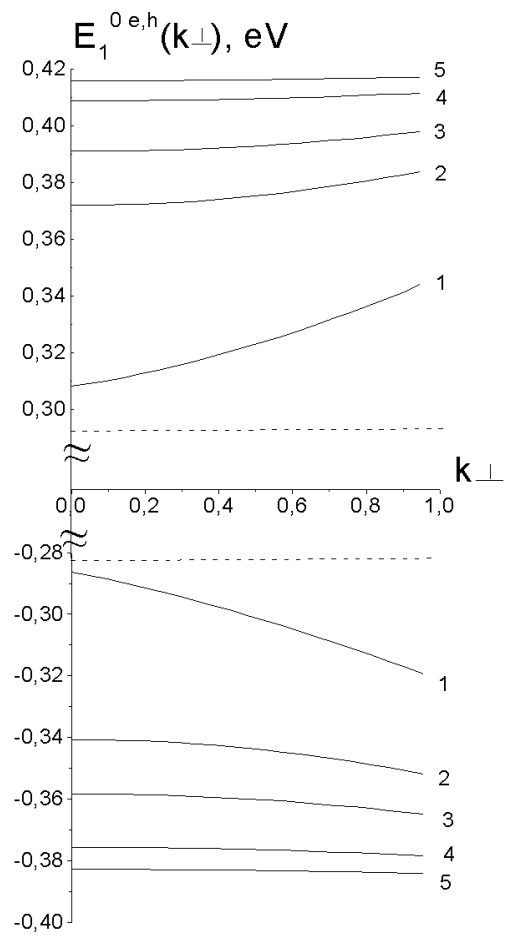

(b)

Figure 2. (a) - Dispersion law of the CSQW quasiparticle ground zone at $b=$ $16 a_{\mathrm{CdS}}$ for the different values of well radius $\rho_{0}: 1-4 a_{\mathrm{HgS}}, 2-6 a_{\mathrm{HgS}}, 3-8 a_{\mathrm{HgS}}, 4-$ $10 a_{\mathrm{HgS}}, 5-12 a_{\mathrm{HgS}} \cdot(\mathrm{b})$ - Dispersion laws of the CSQW quasiparticle ground zone at $\rho_{0}=10 a_{\mathrm{HgS}}$ for the different distances between the wells $b: 1-12 a_{\mathrm{CdS}}, 2-$ $16 a_{\mathrm{CdS}}, 3-20 a_{\mathrm{CdS}}, 4-30 a_{\mathrm{CdS}}, 5-40 a_{\mathrm{CdS}}$

Let us note that in zeroth approximation the longitudinal effective mass does not depend on the $n$ state but in the first approximation it essentially depends on it. If it is necessary, the energy spectrum and wave functions can be further obtained according to the perturbation theory.

\section{Electron and hole spectra in $\beta$-HgS superlattice quantum wires in $\beta$-CdS matrix}

The theory developed is applied for calculating the electron and hole spectra in the rectangular superlattice formed by the $\beta$ - HgS quantum wires embedded into $\beta$-CdS crystal.

Such a system is chosen because both crystals have got a very close size of unit cells and the media parting border between them is very strict (without the transition region) as in the case of complicated quantum wells formed experimentally $[3]$.

The main attention is paid to the analysis of the electron and hole energy spectra $\left(E_{n}^{0 e, h}\left(\vec{k}_{\perp}\right)\right)$ dependencies corresponding to the movement of these quasiparticles in 
the plane $\perp$ to the axial axis of QW because the square dependence of the energies

$$
E_{n}^{e, h}\left(k_{\|}\right)=\frac{\hbar^{2}}{2 \mu_{\| n}^{(1) e, h}} k_{\|}^{2}
$$

on the longitudinal term of qusimomentum is trivial.

The calculation of $E_{n}^{0}\left(k_{\perp}\right)$ was performed according to (17) for the system with parameters given in the table. In figure $2 \mathrm{a}$ the results of calculating the electron

Table 1. Parameters of crystals

\begin{tabular}{rllllll}
\hline medium & $\mu_{e}\left(\mu_{0}\right)$ & $\mu_{h}\left(\mu_{0}\right)$ & $U_{0}^{e}(\mathrm{eV})$ & $U_{0}^{h}(\mathrm{eV})$ & $a(\AA)$ & $E_{g}(\mathrm{eV})$ \\
\hline $\mathrm{CdS}$ & 0.2 & 0.7 & 1.2 & 0.8 & 5.818 & 2.5 \\
$\mathrm{HgS}$ & 0.036 & 0.044 & 0 & 0 & 5.851 & 0.5 \\
\hline
\end{tabular}

and hole ground zones energy dependencies on the QW radius at a fixed barrier thickness are presented. It is clear that the width of the zones is practically not sensitive to the changing of the radius but their location is very strongly (hundreds of meV) shifted into the region of lower absolute energy values at the increasing of QW radius.

In figure $2 \mathrm{~b}$ the results of $E_{1}^{0 e}\left(k_{\perp}\right), E_{1}^{0 h}\left(k_{\perp}\right)$ dependence on the barrier thickness at the fixed QW radius is presented. The figure shows that when the barrier size decreases, both zones essentially (hundreds meV) shift into the region of lower absolute energy values. Herein their width increases a dozen times which is equal to the decreasing of the corresponding effective mass $\left(\mu_{\perp}\right)$ component.

The developed theory of the electron and hole spectra in superlattice quantum wires shows that having changed the spatial size of superlattice quantum wires we can control the fundamental characteristics of quasiparticles in a very wide scale.

\section{References}

1. Alferov G.I. History and future of semiconductor heterostructures. // FTP, 1998, vol. 32, No. 1, p. 3-11 (in Russian).

2. Tsidilkovsky I.M. Electrons and Holes in Semiconductors. Moscow, Nauka (in Russian).

3. Schoos D., Mews A., Weller H. Quantum dot quantum well CdS/HgS/CdS. // Phys. Rev. B, 1994, vol. 49, No. 24, p. 17072-17078. 


\title{
Спектри електронів і дірок у надгратці циліндричних квантових дротів
}

\author{
М.В.Ткач ${ }^{1}$, І.В.Пронишин ${ }^{1}$, О.М.Маханець ${ }^{1}$, Г.Г.Зегря ${ }^{2}$, \\ В.М.Головач ${ }^{2}$ \\ 1 Чернівецький державний університет, \\ вул. Коцюбинського, 2, 274012 Чернівці \\ 2 Фізико-технічний інститут ім. А.Ф.Йоффе, \\ 194021 Ст.Петербург, Росія
}

Отримано 26 червня 1998 р.

Методом приєднаних плоских хвиль розраховані спектри електронів і дірок у надгратці циліндричних квантових дротів $\beta$-HgS у матриці $\beta$ CdS. Складова енрергії, що обумовлена рухом квазічастинок у напрямку перпендикулярному аксіальній вісі дроту являє собою чергування зон з додатньою та від'ємною ефективною масою. Врахування потенціалу надгратки квантових дротів приводить до зняття виродження за магнітним квантовим числом при відході від точки Г зони Брілюена. Досліджені залежності енергій основних зон плоского руху квазічастинок від радіуса квантових дротів і відстані між дротами.

Ключові слова: напівпровідник, електрон, дірка, надгратка, квантовий дріт

PACS: $79.60 . j v$ 